\title{
Flight Simulator Motion Literature Pertinent to Airline-Pilot Recurrent Training and Evaluation
}

\author{
Judith Bürki-Cohen, ${ }^{1}$ Andrea L. Sparko, ${ }^{2}$ and Miles Bellman ${ }^{3}$ \\ USDOT/RITA/Volpe National Transportation Systems Center, Cambridge, MA 02142
}

\begin{abstract}
There has been much debate over the years regarding the need for flight simulator motion for airline-pilot training and evaluation. From the intuitive perspective there is the dictum, "The airplane moves, so the simulator must move" - but intuition alone is not enough to make a valid case for requiring elaborate-and costly-motion systems for training and evaluation of airline pilots. The ultimate goal of training and checking-to produce and maintain highly-skilled pilots capable of ensuring a superior level of safety in both normal and abnormal flight operations-cannot be met without adequate resources available to all airlines world-wide. A big part of the motion debate is what resources are adequate-whether the current regulations specifying six-degree-of-freedom Stewart-motion platforms are appropriate or whether the regulations need to be modified; whether advanced-motion-system simulators, which more realistically simulate aircraft motion, are needed; or whether more flexible motion criteria opening the field for alternative motion stimulation would improve universal access to simulator training and thus benefit safety. While there has been much debate, the main issues regarding motion can be broken down into four main facets: 1 ) how the human perception system responds to airplane motion; 2) the limits of the hexapod-motion systems currently regulated for pilot training in accurately simulating airplane motion; 3 ) the opinions of aviation experts who experience the issue in the real training environment; and 4) empirical evidence on the necessity of physical motion systems for effective simulator training and checking to ensure transfer between simulator and airplane. This paper brings all of these aspects together by summarizing the literature pertinent to the simulator-motion debate. First, we discuss the extent to which humans' ability to perceive and appropriately respond to aircraft disturbances depends on visual motion, physical motion, or both. Second, current limitations in flight data availability for certain scenarios, as well as simulator hardware limitations, are discussed. Third, we summarize and respond to the published positions of several stakeholders. Fourth, a range of analytic and empirical work is summarized, from enhanced motion-feedback modeling to pilot-in-the-loop experiments, as well as meta-analytical work on the benefit of motion simulation on transfer of training to the airplane. This includes a synthesis of the Federal Aviation Administration/Volpe Center empirical studies to date.
\end{abstract}

\section{Nomenclature}

$V_{1} \quad=$ takeoff decision speed; the minimum speed in the takeoff, following a failure of the critical engine, at which the pilot can continue the takeoff and achieve the required height above the takeoff surface within the takeoff distance

$V_{2} \quad=$ takeoff safety speed; a speed that will provide at least the gradient of climb required by the airplane certification rules with the critical engine inoperative

$V_{1} / V_{2}$ cut $=$ engine failure at or above $\mathrm{V}_{1}$ or $\mathrm{V}_{2}$, respectively, with continued takeoff

\footnotetext{
${ }^{1}$ Engineering Psychologist, Human Factors Research and System Applications Center of Innovation, RVT-81, 55 Broadway. Senior Member AIAA.

${ }^{2}$ Engineering Psychologist, Human Factors Research and System Applications Center of Innovation, RVT-81, 55 Broadway. Member AIAA.

${ }^{3}$ Aerospace Engineer, Navigation and Surveillance Division, RVT-71, 55 Broadway.
}

Page 1 of 17 


\section{Introduction}

There has been much debate over the years regarding the need for flight simulator motion for airline-pilot training and evaluation. From the intuitive perspective there is the dictum, "The airplane moves, so the simulator must move" - but intuition alone is not enough to make a valid case for requiring elaborate-and costly-motion systems for training and evaluation of airline pilots. The ultimate goal of training and checking - to produce and maintain highly-skilled pilots capable of ensuring a superior level of safety in both normal and abnormal flight operations - cannot be met without adequate resources available to all airlines world-wide. A big part of the motion debate is what resources are adequate-whether the current regulations specifying 6-degree-of-freedom (DOF) Stewart-motion platforms are appropriate or whether the regulations need to be modified; whether advanced-motionsystem simulators, which more realistically simulate aircraft motion, are needed; or whether more flexible motion criteria, which would open the field for alternative motion stimulation, would improve universal access to simulator training and thus benefit safety. While there has been much debate, the main issues regarding motion can be broken down into four main facets: 1) how the human perception system responds to airplane motion; 2) the limits of the hexapod-motion systems currently regulated for pilot training in accurately simulating airplane motion; 3) the opinions of aviation experts who experience the issue in the real training environment; and 4) empirical evidence on the necessity of physical motion systems to ensure transfer between simulator and airplane. This paper brings all of these aspects together by summarizing the literature pertinent to the simulator-motion debate. First, we discuss the extent to which humans' ability to perceive and appropriately respond to aircraft disturbances depends on visual motion, physical motion, or both. Second, current limitations in flight data availability for certain scenarios, as well as simulator hardware limitations, are discussed. Third, we summarize and respond to the published positions of several stakeholders. Fourth, a range of analytic and empirical work is summarized, from enhanced motion-feedback modeling to pilot-in-the-loop experiments, as well as meta-analytical work on the benefit of motion simulation on transfer of training to the airplane. This includes a synthesis of the FAA/Volpe Center empirical studies to date.

\section{Types of Motion Feedback and How They Are Perceived}

Any discussion of the role of motion cues for pilot training and evaluation in the simulator must be based on the role of motion cues in the airplane. There is wide-spread consensus that correctly-interpreted in-air motion cues provide two types of feedback to pilots (see, e.g., Ref. 1):

a) Maneuver or tracking motion cues provide feedback on the pilot's control inputs, which helps the pilot to keep the airplane on its flight path.

b) Disturbance motion cues alert the pilot that external forces have "disturbed" the flight path and thus increased the tracking error. They help the pilot to initiate the appropriate compensatory control actions.

The next question is how these two types of motion are perceived. Humans perceive self-motion both visually via the photoreceptors in their eyes and non-visually via the mechanical force receptors in their gravito-inertialsomatosensory system. The latter includes the vestibular system in the inner ear, graviceptors in the kidneys, the somatosensory system in the skin and muscles, and even air flow over the skin. ${ }^{2}$ For maneuvering of stable aircraft such as transport airplanes, where all motion does is provide feedback on the pilot-initiated maneuvers, it is generally agreed that visual feedback from out the window (OTW) or from the instruments, which provides the error between intended and actual track visually, is sufficient to make the necessary corrections to stay on track (see, e.g., Ref. 3).

There is some theoretical basis, however, to assume that vestibular motion stimulation is faster recognized than visual stimulation. This may be important for disturbances arising from the world outside the pilot-airplane control loop, such as weather or system failures, where it may be critical for the pilot to immediately decide on and initiate the appropriate control inputs to keep these motions as small as possible. ${ }^{4-6}$ This is because the hair cells in the semicircular canals and in the otoliths of the vestibular system perceive motion directly from the gravito-inertial forces, without pilots having to direct their attention to the stimuli in the external world. ${ }^{7,8}$ To perceive motion via the central visual system, pilots have to direct their attention, either towards the instruments or OTW. But once pilots' attention is focused on the instruments, they receive direct, accurate, and complete information on the airplane's attitude and position status and trends, including also information on the critical airplane systems. OTW, pilots derive motion indirectly from changes in orientation and position of the airplane with respect to the external world. An advantage of vestibular over visual motion perception has been confirmed in the Federal Aviation Administration (FAA)/Volpe Center quasi-transfer studies that are discussed in detail later in this paper-even when 
pilots knew of an impending engine failure, they responded slower when the simulator motion was turned off. ${ }^{\S}$ However, these studies have also shown that: 1) the reaction time difference in transport-category aircraft with motion compared to visual feedback alone was so small that it did not result in more effective control inputs; 2) both experienced and inexperienced pilots were able to use motion cues even without prior training with motion; 3) relatively small excursions provided by a seat placed on a vibrating cockpit floor were enough to alert pilots of disturbances such as engine failures and shifting winds, so that full-motion cueing provided no advantage over seat motion.

\section{Visual and Vestibular Illusions}

Despite the potential reaction time advantage of vestibular motion over visual motion cues, the visual system is generally recognized as "by far the most important sensory system providing spatial orientation during flight." ${ }^{9}$ This is because of the unreliability of the gravito-inertial-somatosensory system, known to represent a great danger for aviators. It leads to spatial disorientation, which is defined as "an incorrect perception of one's linear and angular position and motion relative to the plane of the earth's surface." ${ }^{\prime 9}$ The same reference goes on to explain that such sensory illusions can lead to a difference between instrument indications and what the pilot feels the airplane is doing. In such cases, and "when adequate external visual references are not available," pilots are instructed to fly by the instruments, and warned not to heed the sensations from the "seat of their pants." Any pilot is subject to sensory illusions regardless of experience; the difference between a novice and an experienced pilot is that the latter will know what systems to trust, namely, the instruments.

Earlier, we discussed some differences between the perception of visual information and gravito-inertial forces, observing that visual information has the disadvantage of requiring directed attention while gravito-inertial forces have the advantage of being perceived "omnidirectionally," i.e., regardless of the direction of our attention. Unfortunately, however, only the onset of mechanical forces is perceived reliably. Unlike visual cues that are sensed via chemical processes in the eye that will continue to be relayed to the brain as long as we are looking at them, gravito-inertial forces are perceived via mechanical receptors that habituate to continuous stimulation and stop firing. Pilots may thus habituate to gradual turns or to continuous spins, and then, when a corrective action such as leveling the wings or applying opposite rudder is executed, perceive an uncommanded movement in the opposite direction. This may result in application of control actions that exacerbate the original maneuver and lead to unusual attitudes or even airplane upsets. Such spatial disorientation has led to several loss-of-control accidents. In fact, these are the most common accidents according to world-wide statistics compiled by The Boeing Company. ${ }^{10,11}$ The urgent need for effective training in the prevention, recognition, and recovery from unusual airplane attitudes has therefore been increasingly recognized. ${ }^{12-14}$ Although this discussion should have made it clear that it is critical that pilots rely on their instruments first and foremost, it may be argued that gravito-inertial-somatosensory motion cues are required to train pilots to suppress the temptation to rely on their mechanical motion perception systems. But even if such a need could be empirically documented, none of the simulator motion systems currently employed in pilot training are able to provide the sustained motion cues needed to elicit the somatosensory illusions described above.

\section{Limits on the Quality of Motion}

Many proponents of the need for simulator motion seem to ignore that Stewart-platform motion has severe limitations. These are due to the lack of available validated flight data, and the physical limitations of ground-based motion. It has been documented that some maneuvers lie outside the validated data envelope of today's training simulators, and, even if the data were available, the motions required would lie outside the capabilities of the standard Stewart-hexapod-motion platform used in training. ${ }^{15}$

Airplane motion has complex and rich dynamics even during normal flight. The complexity arises from various nonlinearities affecting the overall motion, while the richness is due to various sources of dynamics that may be excited during flight through the atmosphere. The motion that a pilot experiences inside the cockpit is not only the three-dimensional nonlinear rigid-body motion of the airplane, but also the motion due to the flexible structural dynamics of the airplane. The latter may interact with the rigid-body dynamics. Various kinds of internal or external inputs, such as pilot commands or atmospheric disturbances that the airplane encounters during its flight, may excite all these dynamics at once.

\footnotetext{
${ }^{\S}$ It is unclear whether this is due to the mathematically more direct sensation of gravito-inertial forces compared to OTW visual information, or due to the directional nature of visual perception compared to vestibular and somatosensory perception which does not require directed attention.
} 
If such complex and rich airplane-motion dynamics are to be completely simulated to achieve high physical fidelity, the dynamics model used must include and capture all aspects of the dynamics involved that contribute to the resulting airplane motion. Even in the context of the transport-airplane category, which is the scope of the discussion here and the dynamics of which are considered quite benign in the whole aircraft spectrum, it would be very difficult and costly to build such a complete dynamics model.

Another simplification or modification in the simulation of airplane motion is driven by the motion-system hardware limitations. These hardware constraints have an even stronger impact on the physical fidelity of the resulting motion than the dynamics-model simplification just described. In fact, many of the improvements of the motion platform since the inception of motion-base flight simulation have been in overcoming some of these hardware limitations. These improvements have translated into increased motion-platform capability, be it in the maximum displacement that it can produce or in the frequency bandwidth that it can dynamically cover. Nevertheless, it is questionable whether a ground-based device that is within financial reach of today's airlines will ever be able to provide the sustained motion cues experienced in the air.

\section{Vection or the Visual Illusion of Self-Motion}

Regardless of the limits on the fidelity of ground-based motion, physical motion is purported to also enhance the perceived realism of flight simulation. Realism in simulation is important to elicit in pilot-trainees similar emotional states and responses as in the real airplane. A so-called "simulator mind set," where pilots are too relaxed or even indulge in behaviors that would be unacceptable in the airplane, can be detrimental to training. ${ }^{16,17}$ However, it has been consistently reported that pilots and even instructors are often unaware of the motion state of the airplane (e.g., Ref. 1), as also shown in the FAA/Volpe studies (see section "FAA/Volpe Center Studies"). This is due to the phenomenon of vection, i.e., the illusion of self-motion in the absence of physical motion. It results from the perception of a change in locations (displacement) of optic features or "landmarks" relative to the pilot's position and from optic flow, i.e., the continuous movement of the images of all objects in the environment relative to the viewer. Early studies of vection (e.g., Ref. 18) using striped drums rotating around human subjects found a considerable delay in the onset of vection compared to the perception of motion via the vestibular system. More recently, however, researchers concerned with virtual reality have found a much more powerful and immediate effect with full immersion in a rich and detailed wide-field-of-view (FOV) environment as it is encountered in today's flight simulators. ${ }^{19}$ It has also been found that realistic auditory cues, as they are available in today's simulators, enhance vection. ${ }^{20}$

\section{Opinions on the Need for Motion}

Most of the effort in improving the motion-cue generation is driven by the opinion that better training can be achieved by immersing the pilots in higher motion realism. The airplane moves. Therefore, the airplane simulator has to move. This is and has been a compelling argument for many. Most early flight simulators employed some type of motion, because "[i]t was mistakenly believed during this period that the vestibular apparatus enabled a person to sense orientation in the air as well as on the ground (Robinson, 1973). It was later realized that orientation depends largely on vision” (Ref. 21; see Ref. 14 for an overview of early flight simulators). As instrument flying became more common, however, the realism and training value of these early motion systems was questioned, and fixed-base simulators were preferred over motion simulators. This preference was bolstered by the argument that pilots should fly by instruments rather than by the "seat of their pants." ${ }^{21}$ In fact, as was discussed earlier, perceiving orientation from mechanical sensors (which are prone to desensitization from repeated stimulation) instead of the more robust visual system is inherently dangerous. Although the most prominent early simulator developer, Edward Link, continued to insist that "trainer motion was needed even if incorrect, since motion was present in flying," he eventually developed a fixed-base simulator that sold very well. ${ }^{21}$ Not until the development of the Stewart platform in the mid-1960s, which is moved by six synergistic actuators, did motion systems regain popularity.

\section{A. Joint FAA/Industry Symposium on Level B Airplane Simulator Motion Requirements}

The face value of simulator motion systems was evident in the conclusions reached by a 1996 Joint FAA/Industry Symposium on Level B Airplane Simulator Motion Requirements, which was convened to discuss the need for motion for recurrent airline-pilot training and checking. ${ }^{22}$ The symposium was part of an effort to improve access to flight-simulator training after a slew of commuter-airline accidents in the early 1990s and the ensuing "One Level of Safety" rule. ${ }^{23}$ The 19 subject-matter experts (SME) from industry, academia, and FAA agreed that in the absence of visual reference cues, simulator motion theoretically should provide an early alert for sudden disturbances caused by system failures or weather. They based this on the assumption that vection may be too slow 
to develop to function as an effective alert. This assumption, however, has been weakened by later research on vection discussed above, and by empirical research quantifying the delay in the recognition of flight-path disturbances (see "FAA/Volpe Center Studies"). The experts also conceded that there was a lack of evidence on the effect of motion on transfer of flying skills between the airplane and the simulator, which provided the foundation of the FAA/Volpe Center Flight Simulator Human Factors Program's research on the need for flight-simulator motion discussed later in this paper. The FAA/Volpe Center work was unable to document any clear justification for the requirement of motion, but it has helped trigger much work on closed-loop manual-control models defending the need for motion. This work mostly focuses on how well-trained pilots perform in ideal circumstances during tracking tasks (see sections VII. A and B). Unfortunately, it did not trigger much work on transfer of training. It also triggered some very strong opinion papers from different interest groups, which will be discussed below.

\section{B. ICAO Doc. 9625}

The question of motion was also a central topic in the discussions of the International Working Group (IWG) revising and expanding the international criteria for Flight Simulation Training Devices (FSTD), resulting in Volume I of Edition 3 of the Manual of Criteria for the Qualification of Flight Simulation Training Devices, International Civil Aviation Organization ICAO Document 9625. ${ }^{24}$

Volume I of the manual covers the qualification criteria for airplane FSTD. Part I, "Training Task Derived Flight Simulation Requirements" is based on an analysis of airline-pilot training needs and lists the features (e.g., cues) and fidelity levels required to support each task. Part II, "Flight Simulation Training Device Criteria," lists criteria for standard examples of FSTD derived from Part I, supporting defined training types (e.g., MPL1 - Multicrew Pilot Licence - Phase 1, Core flying skills). Volume I defines seven example FSTD. Part III, "Flight Simulation Feature and Fidelity Level Criteria" serves to define the qualification and validation testing requirements for feature-fidelity levels determined in Part I. It allows operators and manufacturers to design purpose-built devices that do not correspond to any of the standard examples defined in Part II. It also permits currently qualified FSTD to be upgraded to meet new regulations by comparing their features with those defined in Part I.

The challenge of defining objective criteria for the evaluation of motion systems leaves this area subject to further study. Ian Strachan, Fellow of the Royal Aeronautical Society (RAeS) and member of the Flight Simulation Group (FSG), when summarizing the efforts of the IWG, reported that the IWG panel "agreed that the highest level devices would be required for check rides." ${ }^{25}$ He further expressed his concern that some "people and organizations have been proposing that experienced pilots should have recurrency checks on lower-level training devices... without motion platforms." He found this argument "arrogant" because "experienced pilots have accidents too. Therefore, they should have to use the best available training aids...to minimize that possibility." He argued that motion cues "are critical to realistic simulation of turbulence-windshear, downbursts, ...engine failures, instrument, poor visibility and night flying in general" (note that the maneuvers tested in the FAA/Volpe Center motion requirements studies included engine failures in poor visibility and hand-flown one-engine-out landings with microbursts or shifting quartering headwinds-yet failed to demonstrate a benefit of motion). Despite this strong opinion, the Part III provision of a means to evaluate any FSTD based on training task analyses, instead of relying on the technological standards, presumably was intended to give individual National Aviation Authorities (NAA) a means to authorize the use of a new-generation of high-performance fixed-base simulators with a dynamic seat for recurrent training. Recurrent training in such devices has been authorized by at least 10 NAA, of which five additionally permit recurrent operational checking (France, Oman, Fiji, Brazil, United Kingdom). ${ }^{26}$

\section{United States’ Air Line Pilots Association}

The need for motion is fervently supported by the United States' Air Line Pilots Association (ALPA) in a "Safety Committee Statement of Position" ${ }^{\text {,7 }}$ published in September 2007. Referencing interviews with two simulator manufacturers, the paper reports that the vestibular system "leads visual perceived angular rate by approximately $150 \mathrm{~ms}$," and that the "human eyes are limited to approximately a $2 \mathrm{~Hz}$ bandwidth, i.e. a 0.5 second reaction time when it comes to interpreting onset acceleration cues." A first question is whether the vestibular system can indeed directly perceive angular rate or any rates for that matter. The vestibular system consists of the semicircular canals and the otoliths, which both function as mechanical force transducers and thus are only sensitive to angular and linear accelerations, respectively, not rates. Just as "the eye" (in fact, it is the brain that does the processing) has to differentiate velocity from position and acceleration from velocity, "the vestibular system" (and all other mechanical receptors involved in self-motion perception, i.e., the entire gravito-inertial-somatosensory system) has to integrate to get velocity and position from acceleration. The second question refers to the relevance of sensory bandwidth. Any bandwidth would also have to indicate the amount of information processed at $2 \mathrm{~Hz}$, and reaction time would have to include not only the rate of sensory information processing, but also the much slower 
perceptual and cognitive processes leading to an action. All this robs such numbers of meaning in the motion vs. nomotion debate, especially when presented without detailed information on what exactly they mean and how they were derived. But even if we take them at face value, it is unlikely that such small differences would lead to operationally relevant differences in pilot behavior and flight precision for transport aircraft that have, as stated in the ALPA paper, "relatively slow dynamic properties" and, moreover, are designed for maximum stability. Even if such differences were found, they would improbably transfer from the simulator to the airplane. Humans are exercising their use of gravito-inertial-somatosensory information in all aspects of their lives and may not need to be trained to use it. This has been shown empirically in the FAA/Volpe Center quasi-transfer studies discussed later.

The ALPA paper then goes on to describe tracking and disturbance tasks, implying that motion is needed for training both tasks, contrary to commonly held opinion (see above). The section "Salient Research," cites Hall's work $^{28}$ as establishing that "the pilots' acceptance of a simulator for training... was dependent on the presence of motion, even if a wide-field-of-view display system is provided." Hall is also cited as concluding "that motion was especially important for instrument flight." Hall's paper is an unconvincing choice to make these points for several reasons:

a) This 1978 study used motion (3-DOF) and visual systems (head-up display and head-down instruments; television display and skyscape projection for central and peripheral vision, respectively, none described in detail) that have long been superseded by much more advanced systems.

b) The simulated aircraft was a vertical and/or short takeoff and landing (V/STOL) Harrier GR Mk3, which is not representative of fixed-wing transport airplanes.

c) The paper examined the effect of motion when the simulator is used as a tool to assess the handling qualities of aircraft systems. The effect of motion on training or transfer of training was not assessed-in fact, the author states that "[i]n the training field, it is sufficient to show that the overall cost of training is reduced by the use of simulators." (Albeit adding that "in some respects, the pilot adopts an unrealistic control strategy which has to be corrected by flight training.")

ALPA also cites Reid and Nahon, ${ }^{29}$ who, using a B747 simulator with 6-DOF motion and a 40x30-inches-FOV visual system, found an effect of motion only on pilots' opinions, but not on performance and control behavior. ALPA's opinion remains unaffected by Reid and Nahon's evidence that with a transport airplane, even a relatively small FOV visual system appears to generate sufficient vection to elicit the same behavior and flight precision as physical motion. Instead, ALPA focuses on the effect on pilots' opinion. This effect is tempered, however, by a study by Bussolari and Lee, ${ }^{30}$ which found no difference in pilots' ratings of simulator acceptability when the motion status was concealed from pilots. This result was replicated in the FAA/Volpe studies which also concealed the motion status of the simulator and found little evidence that the motion status affected pilots' opinions in the surprisingly few cases it was identified.

Finally, the ALPA white paper also discusses the two earliest FAA/Volpe studies of the requirement for motion, ignoring the 2005 paper $^{31}$ that basically replicates the second study ${ }^{32}$ for initial training and yet another airplane. For the first study, ${ }^{33}$ which trained commuter-airline captains with and without motion in a turboprop simulator and then quasi-transferred them to the same simulator with motion, ALPA reports that no clear conclusions were drawn because of the quality of the lateral acceleration. Well, in fact the conclusion was that there was no effect of motion in an FAA-qualified simulator that was used round-the-clock for training and checking of regional-airline pilots. Its lateral-acceleration motion, although shown to be attenuated compared to the aircraft model, may have been quite typical for many simulators, as found in a follow-up study examining the data of nine other FAA-qualified simulators (follow-up study summarized in Ref. 34). ALPA also cites Go, Bürki-Cohen, Chung, Schroeder, Saillant, Jacobs, and Longridge, ${ }^{32}$ who report that "[f]or recurrent training, no benefit of the motion provided was found." For this study, the motion had been tuned by National Aeronautics and Space Administration (NASA) engineers using the criteria developed by Sinacori, ${ }^{35}$ Schroeder, ${ }^{36}$ and Mikula, Chung, and Tran, ${ }^{37}$ to better represent the airplane for the maneuvers tested. ALPA dismissed these criteria as subjective, although the current efforts in improving motion fidelity are proposing the same plots of phase on the y-axis over gain on the x-axis. ${ }^{38}$ ALPA further criticizes that Go et al. ${ }^{32}$ use steady control inputs as a metric for good piloting. In fact, Go et al. report the metric of higher flight precision achieved with steadier control inputs. Surprisingly, this metric characterized the pilots trained without motion during training and during quasi-transfer to the simulator with motion compared to the pilots trained with motion. Even with this striking difference, the ALPA paper concludes that these results show nothing more than the adaptability of pilots to the presence or absence of motion. Finally, ALPA states that the use of quasi-transfer to the simulator with motion as a stand-in for the airplane, especially with tuned motion, "creates a new primacy of learning for the test subjects in the no-motion versus the pro-motion [sic] group.” Wouldn't it be precisely this argument that should give the motion-trained group an advantage over the group that has to transition from the fixed-base simulator to the unfamiliar motion-base simulator, if motion is indeed an important factor? 
The rest of the paper argues "Why we need motion" based on the fact that the overall increase in aviation safety has reduced pilots' opportunities "to perform abnormal, emergency or extreme maneuvers in the course of...line flying." This is an argument that can just as well be used to support the need for improved simulator access for all pilots. To bolster its thesis that the vestibular system must be stimulated during simulator training and evaluation, ALPA declares that "[o]ther senses (i.e. visual, aural, tactile) are important, but complementary." This section stands in stark contrast with the universally accepted mantra that pilots shall fly by the instruments (see section II of this paper and below). ALPA also underscores the importance of the development of objective motion standards, which is indeed a precondition to any requirement for motion, but even the best of standards alone will not overcome the inherent physical limitations of a Stewart-platform system. Finally, the paper "vigorously disagrees" with any use of non-motion FSTD for recurrent or continuing training and evaluation. ALPA's main argument for this statement is that:

[a] significant portion of the training and evaluation of air carrier pilots centers on extremely detailed instrument flight procedures, conducted with limited to no external visual cueing. [...] To attempt to train without motion will mean that the pilot will, by physical definition, adapt. This adaptation will either distract him from his normal level of performance, lead to performance that is different than in real flight, or it will simply prove that he is adaptable without necessarily proving his proficiency in the true flight environment.

This argument runs counter to the argument of the earlier proponents of instrument training without motion, who disparaged flying by the "seat of [ones] pants" (see above). In that context, it is also important to reiterate that relying on the gravito-inertial-somatosensory system instead of the instruments, due to the mechanical receptors responding to gravity and habituating to prolonged stimulation with concomitant reduction in firing rate, can lead to dangerous perceptual illusions that may lead to airplane upsets. In fact, many pilots will tell you that they had to train themselves to ignore any "seat of the pants" cues, and to learn to rely on the instruments. The question is whether you do need to be exposed to motion cues to learn to ignore them. The evidence from the Go et al. study ${ }^{33}$ seems to indicate otherwise. Pilots that were trained without motion adopted a more successful control strategy requiring fewer inputs that they immediately transferred to the simulator with motion. Also, learning theories that propagate learning without error, especially for skills, would second this result. ${ }^{39}$

\section{United States' Regional Airline Association}

In contrast to the ALPA white paper, a Regional Airline Association (RAA) white paper from March 26, $2008{ }^{40^{* *}}$ sent on April 4 to the FAA's John Allen, Director of Flight Standards Service, argues that access to effective and efficient training tools is paramount in the increasingly complex National Airspace System (NAS), and that use of the full spectrum of training resources, including motion and non-motion systems, would improve the training process. The paper refers to mounting evidence that simulator motion may not increase the effectiveness of flight simulators for training and evaluation, and recommends an operational proof-of-concept test that may support increasing regulatory credit for training provided on simulator platforms without motion. The paper explicitly opposes any regulatory decisions that would bar alternatives to training and checking of pilots with full platform motion. It argues that full platform motion increases the overall rental rate of simulators by $25 \%$, and that reducing the requirement for motion would make advanced training devices more accessible, contributing to the industry's efforts to maintain the highest safety standards.

\section{Empirical Work}

This review of empirical work mainly presents studies performed in the past ten years. For earlier work, refer to Ref. 41. This 1998 review of the pertinent literature concluded that "the findings to date do not solve the [NAAs'] questions regarding the role of platform-motion cueing in [air carrier] pilot training and checking." Despite the fact that some studies had shown benefits of platform motion within the simulator, the benefits of motion had not been shown in the critical case of the transfer of training to the airplane. The review stated that technological advances, industry interest, as well as the lessons learned from previous research provided excellent grounds for readdressing the question of the effect of state-of-the-art platform motion in the presence of state-of-the-art OTW and instrument displays on airline-pilot simulator training and evaluation. Indeed, there has been a huge upsurge in motion research, but it is mainly geared toward qualifying and improving motion (e.g., Ref. 38) or examining how pilots use motion cues in the simulator instead of qualifying motion cues with respect to how well airline pilots transfer skills from simulator training to line flights (see sections A and B, below). The bulk of transfer studies (or, more precisely,

\footnotetext{
${ }^{* *}$ Cited by verbal permission from RAA’s Scott Foose, April 20, 2011
} 
quasi-transfer studies to the simulator with motion as a stand-in for the airplane) have been undertaken by the FAA/Volpe Center Flight Simulator Human Factors Program, which will be described in the section titled "FAA/Volpe Center Studies."

\section{A. “A Cybernetic Approach to Assess Simulator Fidelity”}

Perhaps the most notable of the renewed interests in motion-fidelity requirements is a research program funded by the Dutch Science Foundation and conducted by Delft University of Technology (TU Delft), under the title "A Cybernetic Approach to Assess Simulator Fidelity." ${ }^{22}$ The goal of this program is to "develop a method to objectively and quantifiably assess the extent to which a flight simulator supports real-flight pilot control behavior and, when discrepancies occur, to trace them back to the way the multi-modal stimuli are presented in the simulator." This includes discovering "how pilots adapt their control strategy to what simulators support" and, by examining pilot responses to variations in visual and physical motion cues in a research flight simulator, to determine optimal simulator settings that lead to pilot control behavior in the simulator that matches pilot control behavior in the airplane. Examining the resulting studies, however, it seems that the interest of the cybernetic approach program authors is mainly scientific, using their research to develop tools to assess multimodal control strategies. Nevertheless, because these studies publish pilot-performance and control-behavior results, some of the studies are often erroneously being perceived by the research community as supporting a need for physical motion for flight-simulator training.

The cybernetic approach program started in 2006 and has been slated to last six years. It was born out of a TU Delft research paper written by Steurs, Mulder, and van Paassen in $2004 .{ }^{43}$ The paper compared pilots' performance, control behavior, and opinions in an airplane (the Cessna Citation II jet) to those in its simulator (SIMONA, TU Delft's high-level 6-DOF simulator) under a variety of motion conditions. The results, however, showed only slight differences in pilot control behavior between the flight simulator and the airplane, and between different motion filters ranging from no-motion to full-motion. This is remarkable given the fact that only central visual information was given from primary flight displays. The OTW view was obscured in both the airplane and the simulator. In the conclusion, the authors downplay these results somewhat by stating that the tasks may have been too easy for motion cues to take an effect. However, without OTW, this "visual compensation" would have been done using the central visual information, akin to instruments, and as we have explained earlier, this is exactly what every instructor tries to teach pilots.

The authors also stated that only one of the pilots tested in the airplane was also tested in the simulator. In answer to our query, the principal investigator replied that the reason for the truncated simulator data collection was that in the airplane, they were only able to measure and identify a "lumped" pilot model that did not allow differentiation between pilot control adaptations induced by the visual vs. the vestibular stimulations. For this, they would have to be able to compare pilot control behavior based on tracking vs. disturbance feedback. Without a flyby-wire system to insert a disturbance, Steurs et al.'s pilots received only a tracking signal during data collection in the airplane. Thus, additional simulator testing was abandoned because of the inability to compare disturbance and tracking signal responses in the simulator with the lumped responses collected from the airplane.

After realizing this limitation, the researchers embarked on a series of studies developing strategies and tools to identify multimodal pilot behavior through different mathematical models and forcing functions in the simulator alone. ${ }^{44-47}$ The intent was to identify the models and tools that estimate multiple frequency response functions that permit the identification of the different perceptual modes and their contributions to pilot control behavior. Although some of these models have been published and the Cessna Citation II has now been reconfigured with a fly-by-wire system to add a disturbance signal to the pilot control signal, to date no data has been published showing whether motion cues induce pilot control strategies similar to those in an aircraft. ${ }^{43}$

Such data may indeed help shed some light on the relative roles of different sensory systems, visual or vestibular, for different control tasks, at least for the isolated pitch-tracking tasks examined. Operationally, however, pilots fly tasks that incorporate multiple DOFs, so these results will not be directly applicable to decisions with regard to operational training.

\section{B. Other Simulator-Only Studies}

One of the few recent simulator studies that set out to determine how physical motion cues affect actual airline pilots in a realistic flight environment (e.g., full cockpit replication and OTW visuals) was conducted in the 6-DOF hexapod-based National Aerospace Laboratory (NLR) Generic Research Aircraft Cockpit Environment (GRACE) simulation of a Fokker 100 (100-seat airliner with two fuselage-mounted engines). ${ }^{48}$ This study investigated combinations of roll, sway, and heave motion on pilots' perceptions and control activity during a decrab maneuver. Pilots-Flying perceived no differences in the handling qualities or the motion fidelity of the simulator as it related to 
the different motion combinations. Interestingly, only for sway motion did pilots perceive a difference in the motion strength (compared to no sway), and that difference only appeared when there was also an absence of roll. Unfortunately, this study did not assess flight precision data. It did, however, assess pilot behavior using wheel, pedal, and column rates. Results of this analysis indicated a trend $(\mathrm{p}<0.10$, and this without correcting for the fact that they used multiple dependent tests, which may have removed the trend) for the presence of sway to be associated with lower pedal and column rates, but the largest differences between any combination of sway and other motion axes were approximately 0.25 and $0.15 \mathrm{in} / \mathrm{s}$ for column and pedal, respectively. Nevertheless, this result mirrors evidence from studies conducted by the FAA/Volpe Center ${ }^{31-33}$ (see "FAA/Volpe Center Studies") which found some small but statistically significant control-input differences between pilots during training in a fullflight simulator (FFS) with motion on vs. off. In the Volpe studies, however, all of these differences disappeared once all pilots were tested in the FFS with the motion on, so it is possible that Ref. 48's effects might not transfer either.

In Ref. 48, participants reported feeling some type of physical motion $86 \%$ of the time when in fact no lateraldirectional motion was present. In addition, only one participant could correctly identify the no-motion condition every time. Groen, Smaili, and $\operatorname{Hosman}^{49}$ found similar results, also during a decrab maneuver in a similar - yet generic - transport airplane simulated in the 6-DOF-hexapod NLR Generic Fighter Operations Research Cockpit Environment (GFORCE) simulator with OTW view but only basic F-16 fighter cockpit instruments. In this study, airline pilots perceived increases in motion strength when the motion was on compared to off, but pilots reported feeling motion more than $70 \%$ of the time when absolutely no motion was present in visual meteorological conditions. (Refs. 31, 32, and 33 also found that few pilots ever noticed when the motion was off.) In another study in a 4-DOF simulator, airline pilots flying a rolling takeoff exhibited better pitch performance when stronger roll motion was available, and the pilots felt that full motion was most realistic (on average, a difference of "fair" vs. "good" realism ratings for no-motion compared to full-motion). ${ }^{50}$

There are other studies that, while lacking applicability to training of airline pilots in a realistic flight environment, are worth mentioning. For hovering aircraft, there is some evidence to suggest that combinations of roll/pitch and roll/sway improve performance in a hexapod-motion-based simulator. Nieuwenhuizen, Beykirch, Mulder, and Bülthoff ${ }^{51}$ found combined roll-lateral motion to lead to significantly better performance than nomotion as well as partial-motion (e.g., roll-only). Berger, Terzibas, Beykirch, and Bülthoff ${ }^{52}$ found combined pitch and roll motion to improve performance, but these results were clearly influenced by a no-visuals (black screen) condition in which motion had a large effect compared to no-motion (in conditions with visuals, the motion differences were much smaller: equal to or less than, approximately, $1.25 \mathrm{~m}$ distance from target, $1.5 \mathrm{~m} / \mathrm{s}$ velocity, and 1.25 deg pitch or roll angle). Interestingly, for a similar task in a new robotic-arm simulator with a greater motion space, there were only marginal performance differences found between no-motion and full-motion. ${ }^{53}$ There was no consensus on exactly how motion affects control behavior. ${ }^{\dagger \dagger}$

A few studies have replicated an earlier study by Schroeder (1999), in which experienced helicopter pilots performed a yaw-capture task in NASA Ames' Vertical Motion Simulator. ${ }^{36}$ In Schroeder's study, pilot performance (number of target overshoots) was significantly better with sway motion and marginally better with yaw motion. Pedal rates were also lower when sway was added, but there was no behavioral effect of yaw motion. Grant, Yam, Hosman, and Schroeder ${ }^{54}$ and Ellerbroek, Stroosma, Mulder, and van Paassen ${ }^{55}$ replicated this task in hexapod simulators and found similar results for pilot performance. Grant's results for pedal rate were similar to Schroeder's, albeit not as robust, and Ellerbroek et al. found significant decreases in pedal rate for both sway and yaw. Despite this agreement, however, Ellerbroek et al. note that the effects of sway and yaw on pedal rate were significantly (sway) and moderately (yaw) different between their study and Grant's study. For the most part, pilots in all studies thought that the fidelity was better and that less workload was required when sway or yaw were present.

For tracking and disturbance tasks in helicopters, there is some agreement about the benefits of yaw and sway motion. Grant et al. ${ }^{54}$ found performance improvements resulting from both sway and yaw during a disturbance task and a tracking task. For the tracking task, these improvements were qualified by an improvement only when sway was added to yaw and vice-versa. In other words, the independent effects of both sway and yaw were driven primarily by the addition of the second motion cue. In Ellerbroek et al., ${ }^{55}$ pilots flew a combined tracking and disturbance task, and found similar overall results of sway and yaw, but an interaction of the two suggests that the improvement from yaw was stronger when sway was not present.

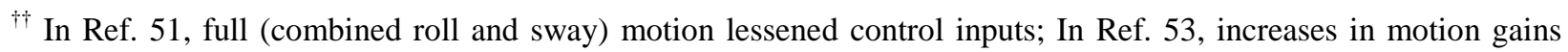
(beyond zero-motion) were associated with decreases in control inputs, but the difference between full-motion and zero-motion was small.
} 
Although simulator-only studies, be they in fixed-wing or rotorcraft, may be interesting theoretically, they do not answer the question of whether behavior or performance differences found in the simulator do transfer to the airplane.

\section{Meta-Analyses of (Quasi) Transfer Studies}

Before moving to the FAA/Volpe Center studies, we will briefly review two meta-analyses focusing on transfer studies. Vaden and Hall analyzed seven transfer studies and found "a small, positive effect on pilot training transfer, $d=0.16$." This effect was too small, however, to be statistically significant. ${ }^{56}$ The paper only included the first FAA/Volpe study, ${ }^{33}$ and claimed that it gave only p-values and no effect sizes (in fact, the study report presents very clear graphs where the actual effect sizes could easily have been read, especially when looking at the graphs in Ref. 33). Also, the paper ignored the second FAA/Volpe study. ${ }^{32}$ All other studies are from before 1982, with one exception: Ref. 57 by Van der Pal from NLR found no beneficial effect of motion in a 6-DOF dome F-16 training simulator in a quasi-transfer study using experienced pilots. But Vaden and Hall state that for this study, "[o]nly partial univariate F values" were available; in fact, also for this study, there was a more extensive report available that might have included the necessary information. The task was a "low-drag weapon delivery." Van der Pal measured flight precision and stick inputs. Pilots were told to focus on the flight parameters, not on the hits. The motion platform was either on or off, but the g-seat and g-suits were on for all subjects.

A more comprehensive meta-analysis was undertaken by Ref. 58, De Winter, Dodou, and Mulder. This study included 24 studies, including the FAA/Volpe studies described in Refs. 32 and 33. It excluded the FAA/Volpe study described in Ref. 31 because it reported the significant differences only. The meta-analysis therefore included only four studies dating from 1998 or later, with some of the remaining studies dating as far back as the 1960s. The authors included only whole-body motion in the study, but with studies spanning four decades, the physical motion systems included in the meta-analysis must have varied greatly. This must have been all the more true for the visual and sound systems as well as the instruments indicating aircraft state, if they were at all present; no details are given as to the other cueing systems contributing to motion perception. Although the authors are to be commended for treating experiment design (transfer; quasi-transfer to the same motion as used in training; quasi-transfer to "better" motion than used in training), type of task (fixed-wing airplane maneuvering; correcting for disturbance in fixedwing airplanes; helicopter flight), and experience level (novice, intermediate, and expert pilots) as moderator variables, they are still comparing apples with oranges; even using a random-effects model will not compensate for such heterogeneity in all systems critical to pilots' perception of motion.

Nevertheless, the relative effect sizes of this otherwise carefully conducted meta-analysis are quite interesting. As is usual in meta-analysis, De Winter et al. report Cohen's effect sizes d; in general and in highly relative terms, an effect size of up to 0.3 is considered a small effect, 0.5 a medium effect, and any effect sizes higher than 0.8 are considered large. ${ }^{59}$ Perhaps the most interesting result when considering equipment needs for recurrent training is that for experienced pilots, the effect of physical motion was calculated to be nil, i.e., $d=-0.01$. This result flies in the face of the strong convictions expressed in Refs. 27 and 25 that physical motion is especially, if not most, important for recurrent pilot training. The lack of an effect for experienced pilots is especially interesting because the authors report that the experiments using experienced pilots tended to use disturbance tasks, for which pilots should rely more heavily on physical motion cues. This increased diagnosticity, of a need for physical motion, of maneuvers that require compensation for external disturbances over maneuvers where motion provides feedback on pilots' intended control actions was in fact confirmed; no significant effect size was found from maneuver motion (d=0.07) and a "large" effect from disturbance motion $(d=0.84)$. The effect size for the helicopter task $(d=0.86)$ confirmed the contention that physical motion is more important when learning how to maneuver unstable aircraft (see Ref. 5). A final interesting result is that the effect sizes were found to be largest for quasi-transfer experiments transferring to identical motion ( $\mathrm{d}=1.19)$, as they were done in the FAA/Volpe Flight Simulator Human Factors Program, and smallest for true-transfer experiments, for which no significant effect was obtained $(\mathrm{d}=0.10)$. This certainly can serve as a counter argument for those who would like to question the validity of the FAA/Volpe studies on the basis that they used the simulator with physical motion as a stand-in for the airplane.

\section{FAA/Volpe Center Studies}

A series of four motion studies have been conducted by the Volpe Center, under the FAA-sponsored Flight Simulator Human Factors Program, in an effort to provide empirical support for or against the necessity of hexapod motion for airline-pilot training. The ultimate success of pilot training was determined by how well the pilots flew the "airplane" after training. If training with simulator motion benefits pilots once they transfer to the airplane, then pilots trained with motion should perform better than pilots trained without it. These studies also asked whether 
training with motion was more or less difficult - i.e., whether or not motion training required more or less work than training without it. In general, these studies did not find a relevant benefit of motion.

In all four studies, two groups of pilots were trained in the simulator - one group in an FFS with the motion on and the other in the same FFS with the motion off (or, in the case of the fourth study, ${ }^{60}$ a training device with an alternative motion system). Both groups of pilots were then quasi-transferred to the FFS with the motion on - a stand-in for the airplane. The quasi-transfer design allowed for greater experimental control because extraneous variables (such as weather) could be held constant for all pilots. With greater experimental control, these studies also had more power to find an existing effect, i.e., it was less likely that an actual effect would be masked by nuisance variability. Moreover, this design allowed the pilots to fly extremely difficult maneuvers which would be dangerous to fly in the airplane; these maneuvers were carefully selected because they were determined, by SME and the literature, to be particularly diagnostic of a need for motion. These were closed-loop maneuvers with sudden disturbances that required immediate action from the pilots. The theoretical basis for the assumption of increased diagnosticity of disturbance tasks is that physical motion cues would serve as an early alert that should benefit pilot response (see section II, “Types of Motion Feedback and How They Are Perceived”).

The first two studies tested recurrent pilots, who, according to the literature (Ref. 61, but see also Ref. 58), have become accustomed, during training and practice, to rely on motion cues. The first study ${ }^{33}$ was done with regionalairline captains in a Level-C FFS simulating a turboprop "powerhouse" airplane with wing-mounted engines. Pilots were first-look evaluated, trained, and then tested on two engine failures on takeoff: rejected takeoffs (RTO) and $\mathrm{V}_{1}$ cuts. Both maneuvers were flown at dusk with low visibility and with a 10-knot crosswind. Very few operationally relevant effects of motion were found, so the researchers wondered if the lack of findings was due to a discrepancy between the simulator's lateral acceleration and that of the flight model (although, in a follow-up study, summarized in Ref. 34, the motion system of the study simulator was found to be equivalent to the motion systems of other similar FFS).

Nevertheless, a second study ${ }^{32}$ endeavored to improve the FFS motion system to see if any effects emerged. With technical and financial help from NASA, the motion system of its Level-D 747-400 FFS was reengineered to improve lateral acceleration, trading off rotational motion which some studies suggest is less important (e.g., Ref. 36). In the second study, ${ }^{32}$ the RTO was replaced with a $V_{2}$ cut, which was argued to be a more motion-dependent maneuver requiring multiaxis control, and two hand-flown engine-out landing maneuvers were added. Takeoffs were flown in daylight conditions with low visibility and a constant 10-knot tailwind. The landing maneuvers were: 1) an instrument landing system (ILS) landing with 10-to-12-knot quartering winds shifting from a headwind to a tailwind, and 2) a sidestep landing (SSL) with a microburst applied on the runway rollout. With the enhanced motion system, small yet statistically significant motion-group differences were found; however, these differences were operationally irrelevant. Arguing that the lack of motion benefits could be due to experienced pilots' familiarity with airplane motion (which is in line with Ref. 58), a third study ${ }^{31}$ was conducted with initial pilots (in an FFS simulating a Boeing 717-200). Here, pilots again flew the $\mathrm{V}_{1}$ cut and the ILS landing, with weather and maneuver details similar to those of the second study. ${ }^{32}$ Again, the results of the third study ${ }^{31}$ mostly confirmed that of the previous one.

Given that so few relevant effects of motion were found across all three studies, a fourth study ${ }^{60}$ evaluated the training effectiveness of an alternative motion system called the Full-Flight-Trainer- $\mathrm{X}^{\mathrm{TM}}$ (FFT-X, or FFT), which had been developed with reference to the earlier studies. The FFT has a high-level visual system and dynamic seats to provide motion simulation; each seat is equipped with electric actuators to provide small-magnitude heave motion; loud speakers under the cockpit provide vibration cues. In this study, ${ }^{60}$ low-experience pilots trained in either the FFT or an FFS simulating a small turboprop airplane, and then all pilots quasi-transferred to the FFS. Pilots flew $\mathrm{V}_{1}$ and $\mathrm{V}_{2}$ cuts, the ILS approach/landing, and the SSL (although, most of the SSL data was not analyzed/reported due to technical problems with data collection).

\section{Takeoffs with Engine Failure}

Directional Performance. For the RTO in Study $1,^{33}$ the only group difference found was an initial directional performance advantage (less yaw activity) for the motion group when they were first evaluated in the FFS (after flying the airplane as captains for 12 months). This difference disappeared once both groups transferred to the simulator with motion turned on. When pilots flew the $\mathrm{V}_{1}$ cut, however, the directional performance was in favor of the no-motion group, who had less yaw activity at transfer, even though this difference was small (on average, less than $0.5 \mathrm{deg} / \mathrm{s}$ ) and it did not affect heading (also note that this difference did not appear during training). The nomotion group's lower yaw activity was accompanied by fewer pedal reversals at transfer (again, the difference was small; less than one reversal). The motion-trained group actually got worse in their heading deviation compared to itself between training and transfer, even though this was not enough to bring about a group difference in heading at transfer. 
With the enhanced motion in Study $2{ }^{32}$ the motion-trained pilots had significantly less yaw activity than the nomotion pilots at evaluation and at training. This was associated with more frequent but smaller-magnitude pedal inputs. The lower yaw activity for the motion group did result in a trend for less heading deviation, but during evaluation only. Once trained, the yaw and heading differences disappeared and did not return at transfer. By transfer, the no-motion pilots managed to adjust their pedal inputs to match those of the motion group, which may have contributed to their directional performance improvement. ${ }^{\neq}$Also in Study $2{ }^{32}$ with enhanced motion, there was a pedal reaction time advantage for the motion group; pilots trained with motion responded an average of 0.4 and 0.3 seconds faster to the engine failure during evaluation and training, respectively. This was not surprising, as the motion was expected to give an early alert to the engine failure. Once both groups transferred to motion, however, the reaction time advantage disappeared; pilots trained without motion were just as capable of taking advantage of the motion alert as the pilots trained with motion, as evidenced by a significant improvement in the nomotion group's pedal reaction time from evaluation to transfer. The pedal reaction time advantage emerged also (but as a trend only) in Study $3^{31}$ with initial pilots, this time with the motion pilots responding 0.47 seconds faster during training. This advantage, however, again disappeared once both groups transferred to motion (again, no-motion pilots significantly improved from training to transfer). For the $\mathrm{V}_{2}$ cut in Study $2,{ }^{32}$ motion-trained pilots actually had a slower reaction time, but only at the transfer stage, so it was probably due to fatigue (this was the only significant performance effect for the Study $2^{32} \mathrm{~V}_{2}$ cut). In the fourth study, ${ }^{60}$ which replaced no-motion training in the FFS with training in the FFT with seat motion, FFS-trained pilots used larger pedal inputs than the FFT-trained pilots during training for the $\mathrm{V}_{1}$ cut, but this did not result in any directional performance differences and, moreover, it did not transfer when both groups flew in the FFS. In fact, both groups of pilots improved their yaw and heading between training and transfer (and to the same degree), regardless of how they worked the pedal during training in the different simulators. For the $\mathrm{V}_{2}$ cut, FFS-trained pilots had less yaw activity during training, but this was not associated with any differences in control activity and it did not transfer.

Longitudinal Performance. For the first study ${ }^{33} \mathrm{~V}_{1}$ cut, the motion-trained group had better airspeed compliance with more pitch activity, and these differences only emerged at transfer. The lower airspeed exceedance was a result of an improvement for the motion-trained group but not for the no-motion group from evaluation to transfer (motion and no-motion pilots had the same average airspeed exceedance at first-look evaluation). The results of Study $3^{31}$ with initial pilots confirmed these differences in airspeed and pitch (although the latter was only a trend, $\mathrm{p}<0.10$ ), except that the differences emerged at training and persisted at transfer, and there was no significant improvement for either group. Motion-trained pilots in Study $3^{31}$ achieved their better airspeed compliance and marginally greater pitch activity with smaller column inputs during both training and transfer. The overall group difference in column inputs was small, however (0.16 inch difference in root-mean-square (RMS) column position), as were the pitch excursions in both studies (overall group difference of 0.38 deg standard deviation (STD) in Study 3 and 0.69 and 0.61 deg STD in Study 1 during evaluation and transfer-testing, respectively). In Study $2,{ }^{32}$ with enhanced motion, the motion-trained pilots actually had less pitch activity than the no-motion pilots, but this effect only emerged during evaluation and did not appear at training or at transfer. This difference in pitch activity did not result in any group differences in airspeed compliance. For the $\mathrm{V}_{2}$ cut in Study $2,{ }^{32}$ the motion group had larger column inputs at transfer, but this effect did not affect performance and did not emerge at evaluation or training, so it may be a result of fatigue. In the fourth study ${ }^{60}$ with training in the FFT, FFStrained pilots had smaller column inputs than the FFT-trained group during training for both $V_{1}$ and $V_{2}$ cuts, but the differences disappeared when both groups transferred to the FFS. Once transferred, the FFT-trained pilots lowered the magnitude of their inputs to match that of the FFS-trained group. Given this, as well as the fact that no other longitudinal control or performance differences were found, the difference in column inputs in the fourth study ${ }^{60}$ may have been due to idiosyncratic differences between the two simulators (note that this is the only study where two different simulators were compared).

Lateral Performance. There were very few effects on lateral performance for takeoff maneuvers. In fact, the only motion effect found was for lateral control-input behavior, and it had no effect on performance. This effect was found for the first study ${ }^{33} V_{1}$ cut, where pilots trained with motion had fewer wheel reversals than the no-motion group at transfer. This difference was small (less than one reversal in the 15 seconds following engine failure), and it did not appear during training.

\footnotetext{
抹 Although no significant performance improvement was evident for either motion group between evaluation and training, the group difference in heading and yaw performance that was present at evaluation had disappeared by training. At training, however, there was still a significant group difference in pedal inputs; they had in fact not changed much since evaluation. This may be evidence to suggest that the small group differences in control inputs found in these studies are not operationally large enough to lead to any significant performance difference.
} 
Summary. Only one of the four studies found any group differences in directional performance once transferred. This was the first study ${ }^{33}$ with recurrent pilots where, in fact, pilots trained without motion performed better, but it could be argued that the simulator's lateral acceleration was substandard, albeit it was found to be typical. With enhanced lateral acceleration in Study $2{ }^{32}$ motion-trained pilots did experience an initial benefit in directional performance, but it did not ultimately impact their performance. No-motion pilots did respond slower with the pedal to the engine failure during training for the $\mathrm{V}_{1}$ cut, but this disadvantage did not transfer, showing that pilots did not need to be trained with motion in order to benefit from the alert. Interestingly, the vibration cues in the FFT seemed to be enough to make up for this no-motion disadvantage, as no pedal reaction time differences were found in the fourth study. ${ }^{60}$ Motion-trained pilots were also found to control their airspeed better in some cases, but they did so with larger pitch excursions. There were some motion-group differences in control inputs, but these rarely affected performance and did not always transfer. Moreover, all of these control-input differences were small and are not considered operationally relevant.

\section{Engine-out Landings}

Directional Performance. Engine-out landings were introduced in Study $2^{32}$ with enhanced motion. This study found better directional performance (less heading deviation) for the no-motion group than for the motion group during the ILS approach and landing, and this effect did transfer when both groups flew the FFS with motion on. This pattern also occurred during the first part of the SSL, but once pilots reached decision height all differences in flight performance disappeared. For the ILS, the better heading for the no-motion group was accompanied by more frequent pedal inputs. For the SSL, only near touchdown (when the microburst occurred) did the no-motion group use more frequent pedal inputs, and this time it did not result in any performance benefit. In Study $3^{31}$ with initial pilots, motion-trained pilots had larger pedal inputs during the ILS prior to reaching decision height which did transfer (the overall group difference was small, however: 0.08 inches RMS), but this effect did not emerge during the touchdown phase. FFS-trained (vs. FFT-trained) pilots in Study $4^{60}$ also had larger pedal inputs during the ILS approach which did transfer, but this difference disappeared by the time pilots reached the touchdown phase.

Longitudinal Performance. Between decision height and touchdown, no-motion pilots in Study $2^{32}$ flew with better airspeed compliance which did transfer, and this performance was coupled with more frequent column inputs. In Study 4, ${ }^{60}$ FFT-trained pilots used larger-magnitude column inputs during training, but this did not transfer to the FFS, nor did it affect any longitudinal performance measures. An effect that emerged in Study $4^{60}$ was that FFStrained pilots had better glideslope compliance, but only during the approach phase (prior to reaching decision height). Also, this effect did not transfer once both groups flew the FFS. No longitudinal effects emerged during the sidestep landing in the two studies that successfully examined side step landings.

Lateral Performance. In Study 2, ${ }^{32}$ no-motion pilots had better localizer compliance and less bank during the approach phase for both the ILS and the SSL, and this did transfer. (The difference in localizer compliance for the ILS (0.19 dot STD) was, according to SME, the most potentially operationally relevant effect for landings.) Nomotion pilots achieved this performance with smaller wheel inputs and fewer wheel reversals. The differences in wheel inputs persisted into the landing phase of the ILS and into latter parts of the SSL (through breakout of clouds to vertical gust, when the sidestepping occurred), although this time there was no resulting performance benefit. In Study $4^{60}$ with the alternative motion system, it was the FFS pilots who exhibited a trend for overall less wheel activity (smaller inputs) during training for the ILS approach, but this effect was diminished at transfer and it was not associated with any group differences in performance. By the touchdown phase, the FFT pilots had lower wheel activity at training, but this reversed at transfer when they increased their wheel activity and the FFS pilots decreased their activity, resulting in smaller inputs for the FFS pilots (significant interaction, but neither of these differences alone were statistically significant). The FFS pilots' decrease in wheel inputs from training to transfer was associated with a significant improvement in localizer compliance at transfer, while the FFT pilots' increase in inputs resulted in no significant change in flight precision.

Touchdown Performance. During touchdown for the second study ${ }^{32}$ SSL, motion-trained pilots landed more softly, but less precisely, than no-motion-trained pilots, and this effect did transfer. In Study $4,{ }^{60}$ a similar effect was found for touchdown during the ILS, with FFS-trained pilots landing more softly than FFT-trained pilots, except this time it did not transfer. What is interesting, however, is that this pattern was reversed when Study $4^{60}$ pilots flew the SSL; this time, FFT-trained pilots flew more softly than FFS-trained pilots, although this effect did not transfer.

Summary. Pilots trained without motion in Study $2^{32}$ showed some advantages in heading, airspeed, and localizer compliance which did transfer; these performance benefits were accompanied by different control strategies (although these differences were small and, for the most part, operationally irrelevant). Potentially the most, or only, operationally relevant difference was the better localizer compliance for the no-motion group, at least according to one SME. In general, motion-trained pilots landed more softly but less precisely than the no-motion pilots, an effect which did transfer for both ILS and SSL in Study $2^{32}$ but not in Study $4 .{ }^{60}$ 


\section{Pilot Perceptions}

Overall, there were very few differences in how pilots perceived themselves and/or the simulators. In general, there were a few group differences in how pilots perceived their own performance/technique and ease of gaining proficiency, but these differences were often only trends and were not consistent across studies. Only in the third study $^{31}$ did any group differences emerge in ratings of the acceptability of the simulator as a stand-in for the airplane; motion-trained pilots in Study $3^{31}$ felt the acceptability of the simulator was better during training, and this almost transferred when both groups flew the simulator with motion on. In some cases, pilots in the no-motion group felt that the simulator cues were worse than pilots in the motion group did, although neither group thought the cues were as good as in the airplane. Interestingly, when asked to freely comment on their experience in the simulator, very few pilots even mentioned the motion status, suggesting that most pilots did not even notice whether the motion was on or off. Pilots did not experience simulator sickness in either motion configuration, nor did they perceive any differences in workload.

\section{Conclusion}

As the FAA launched the "One Level of Safety"23 initiative for major and regional airlines and more and more airlines adopted Advanced Qualification Programs (AQP) in the mid-nineties, the FAA initiated the FAA/Volpe Flight Simulator Human Factors Program to identify potentially unnecessary obstacles to universal access to the benefits of flight-simulator training. A review of the literature and consultation with many SME, including an FAA/industry workshop, ${ }^{22}$ revealed that while simulator motion does have great face value (after all, the airplane moves), decades of research have failed to show an effect of motion on transfer of training to the airplane or on reverse transfer from the airplane to the simulator for pilot evaluation. ${ }^{41}$ The program thus initiated the series of carefully controlled studies described above to answer the following questions: 1) Are there maneuvers in airlinepilot training where platform motion cues, in addition to the visual cues from a wide-FOV OTW view and instruments, result in an operationally relevant improvement of transfer between the simulator and the airplane? 2) Do airline pilots need to be trained to avail themselves of motion cues? 3) Are motion cues from a hexapod platform representative of those experienced in the airplane? 4) Can alternative systems provide onset cues and perception of realism?

To obtain valid and replicable answers to these questions, we did everything possible to ensure the statistical power to find an effect of motion, if there was one. For the first three studies, we tested the extremes; that is, we compared FAA Level-C or D FFS with the motion turned on or off. We calibrated all cueing and measurement systems. We counterbalanced across groups any other factors that we could not keep constant on all aspects that could affect their flying skills. We also tested more pilots than in most other studies to randomly balance any differences that we were unable to systematically control or counterbalance. We used the most diagnostic maneuvers within and even somewhat beyond the air-transport-pilot curriculum that should have depended on an alerting function of physical motion. We used the most diagnostic participants. We measured nearly 80 dependent variables directly from the simulator representing pilot control inputs, flight precision, and simulator-motion performance at a high sampling rate (at least $30 \mathrm{~Hz}$ ). We also asked all participants for their opinions on all aspects of the simulator and the behavior of the pilots flying. We kept the motion status and in fact the purpose of the study secret to prevent any bias for or against motion from affecting the results. To ensure generalizability of the results, we tested three very different groups of airplanes, ranging from an agile and slightly overpowered 31-passenger turbopropeller airplane to a somewhat sluggish jumbo jet, including airplanes with fuselage- and wing-mounted engines. Participants' prior experience was equally diverse, including aspiring first officers on Boeing 717 straight from the school house to Boeing 747 captains with thousands of hours in type.

The results of the first three experiments represent strong evidence that the answers to the first three research questions are "No." Finally, the last question appears to have been answered by the successful training of very lowtime pilots with as little as 500 hours in a high-level simulator with seat motion and vibration only in the fourth study. This simulator is already helping smaller airlines all over the world to provide better training to their pilots by improving their access to a qualified device, which is critical in view of the predicted shortages of pilots with sufficient threat and error management and manual flying skills.

\section{Conclusion}

It is up to the industry and the regulators to decide whether the benefits of full 6-DOF hexapod motion found to date warrant the exclusion of pilot populations all over the world from the benefits of frequent exposure to an affordable home-based device. It appears that generations of pilots have been effectively trained not only procedurally, but also in stick and rudder skills using motion-based FSTD where the motion had been neither rigorously defined nor tested. Much effort is currently underway to improve this situation, which is likely to further 
increase the cost of acquisition, qualification, and maintenance of motion systems. Given the impending pilot shortage, the lower and lower prior experience of new hires, and the increased operational complexity to be expected from next generation air traffic management systems, the wise allocation of training dollars and time will critically affect the safety of the flying public. It may be the design of the entire training program, rather than the individual devices, that will have the largest impact.

\section{Acknowledgments}

The authors thank Dr. Eleana Edens from the FAA Human Factors Research and Engineering Division and Christopher MacWhorter from the FAA Voluntary Safety Programs Branch for helpful comments and support of this work.

\section{References}

${ }^{1}$ Caro, P. W., "The Relationship between Flight Simulator Motion and Training Requirements," Human Factors, Vol. 21, No. 4, 1979, pp. 493-501.

${ }^{2}$ Harris, L. R., Jenkin, M., Zikovitz, D., Redlick, F., Jaekl, P., Jasiobedzka, U., Jenkin, H., and Allison, R. S., "Simulating Self Motion I: Cues for the Perception of Motion," Virtual Reality, Vol. 6, 2001, pp. 75-85.

${ }^{3}$ Levison, W. H., and Junker, A. M., "A Model for the Pilot's Use of Motion Cues in Steady-State Roll-Axis Tracking Tasks," AIAA Flight Simulation Technologies Conference, AIAA, 1978.

${ }^{4}$ Hall, J. R., "The Need for Platform Motion in Modern Piloted Flight Training Simulators," Royal Aerospace Establishment, Tech Memo FM 35, Bedford, UK, 1989.

${ }^{5}$ Gundry, J., "Man and Motion Cues," Third Flight Simulation Symposium, Royal Aeronautical Society (RAeS), 1976.

${ }^{6}$ Hosman, R. J. A. W., and Van der Vaart, J. C., "Effects of Vestibular and Visual Motion Perception on Task Performance," Acta Psychologica, No. 48, 1981, pp. 271-287.

${ }^{7}$ St George, R. J., and Fitzpatrick, R. C., "The Sense of Self-Motion, Orientation and Balance Explored by Vestibular Stimulation," Journal of Physiology, Vol. 589, No. 4, 2011, pp. 807-813.

${ }^{8}$ White, A. D., and Rodchenko, V. V., "Motion Fidelity Criteria Based on Human Perception and Performance," AIAA Modeling and Simulation Technologies Conference, AIAA, 1999.

${ }^{9}$ U.S. Air Force, Air Force Manual 11-217: Instrument Flight Procedures. 2000.

${ }^{10}$ Boeing, Statistical Summary of Commercial Jet Airplane Accidents: Worldwide Operations 1959-2010. 2011, Aviation Safety, Boeing Commercial Airplanes.

${ }^{11}$ Civil Aviation Authority, "Safety Regulation Group Safety Plan," CAA, West Sussex, U.K., 2006.

${ }^{12}$ Advani, S. K., Schroeder, J.A., and Burks, B., "What Really Can Be Done in Simulation to Improve Upset Training," AIAA Modeling and Simulation Technologies Conference, AIAA, 2010.

${ }^{13}$ Bürki-Cohen, J., "Technical Challenges of Upset Recovery Training: Simulating the Element of Surprise," AIAA Modeling and Simulation Technologies Conference, AIAA, 2010.

${ }^{14}$ Bürki-Cohen, J., and Sparko, A. L., "Airplane Upset Prevention Research Needs," AIAA Modeling and Simulation Technologies Conference, AIAA, 2008.

${ }^{15}$ Boeing, Airbus, and Flight Safety International (FSI), Airplane Upset Recovery Training Aid Revision 2, edited by C. D. Carbaugh, C. L. Rockliff, and B. Vandel, 2008.

${ }^{16}$ Bürki-Cohen, J., "Evidence for the Need of Realistic Radio Communications for Airline Pilot Simulator Training and Evaluation," International Conference Simulation of the Environment, Royal Aeronautical Society (RAeS), 2003.

${ }^{17}$ Davies, D. P., "Approval of Flight Simulator Flying Qualities," The Aeronautical Journal, Vol. 79, No. 775, 1975, pp. 281297.

${ }^{18}$ Young, L. R., Dichgans, J., Murphy, R., and Brandt, T., "Interaction of Optokinetic and Vestibular Stimuli in Motion Perception," Acta Otolaryng, Vol. 76, 1973, pp. 24-31.

${ }^{19}$ Riecke, B. E., Västfjäll, D., Larsson, P., and Schulte-Pelkum, J., "Top-Down and Multi-Modal Influences on Self-Motion Perception in Virtual Reality," 1st International Conference on Virtual Reality, of the 11th International Conference on Human Computer Interaction (HCI International), 2005.

${ }^{20}$ Riecke, B., Schulte-Pelkum, J., Caniard, F., and Bülthoff, H., "Spatialized Auditory Cues Enhance the Visually-Induced Self-Motion Illusion (Circular Vection) in Virtual Reality," Max Planck Institute for Biological Cybernetics, Technical Report No. 138, Tübingen, Germany, 2005.

${ }^{21}$ Rolfe, J. M., and Staples, K. J. (eds.), Flight Simulation, Cambridge Aerospace Series, Cambridge University Press, Cambridge, UK, 1986.

${ }^{22}$ Bürki-Cohen, J., Transcript of the Joint FAA/Industry Symposium on Level B Airplane Simulator Motion Requirements, FAA, 1996.

${ }^{23}$ Federal Aviation Administration (FAA), Commuter Operations and General Certification and Operations Requirements in Code of Federal Regulations, Title 14, Parts 91, 119, 121, 125, 127, and 135. 1995.

${ }^{24}$ International Civil Aviation Organization (ICAO), "Manual of Criteria for the Qualification of Flight Simulators, Revision 3," ICAO, 9625-AN/938, Montreal, 2009. 
${ }^{25}$ Strachan, I., "Rationalizing the Regs - RAeS Plays Pivotal Role in Sim Training," Journal for Civil Aviation Training (CAT), 2008, pp. 22-24.

${ }^{26}$ Bigarré, J., and Dranfield, M., “Continuous Pilot Training, Urgencies and Realities - What Are We Waiting For?,” ICAO Next Generation of Aviation Professionals Symposium, ICAO, 2010.

${ }^{27}$ Air Line Pilots Association (ALPA), Safety Committee Statement of Position: The Need for Motion in Flight Simulation. 2007.

${ }^{28}$ Hall, J. R., "Motion versus Visual Cues in Piloted Flight Simulation," Flight Mechanics Panel Specialists' Meeting on Piloted Aircraft Environment Simulation Techniques, Advisory Group for Aerospace Research and Development (AGARD), 1978.

${ }^{29}$ Reid, L. D., and Nahon, M. A., "Response of Airline Pilots to Variations in Flight Simulator Motion Algorithms," Journal of Aircraft, Vol. 25, No. 7, 1988, pp. 639-646.

${ }^{30}$ Bussolari, S. R., and Lee, A. T., "The Effects of Flight Simulator Motion on Pilot Performance and Simulator Acceptability in Transport Category Aircraft," Massachusetts Institute of Technology/NASA Ames Research Center, 1986.

${ }^{31}$ Bürki-Cohen, J., and Go, T. H., "The Effect of Simulator Motion Cues on Initial Training of Airline Pilots," AIAA Modeling and Simulation Technologies Conference, AIAA, 2005.

${ }^{32}$ Go, T. H., Bürki-Cohen, J., Chung, W. W., Schroeder, J., Saillant, G., Jacobs, S., and Longridge, T., "The Effects of Enhanced Hexapod Motion on Airline Pilot Recurrent Training and Evaluation," AIAA Modeling and Simulation Technologies Conference, AIAA, 2003.

${ }^{33}$ Go, T. H., Bürki-Cohen, J., and Soja, N. N., "The Effect of Simulator Motion on Pilot Vehicle Training and Evaluation," AIAA Modeling and Simulation Technologies Conference, AIAA, 2000.

${ }^{34}$ Bürki-Cohen, J., Go, T. H., Chung, W. W., and Schroeder, J., "Simulator Platform Motion Requirements for Recurrent Airline Pilot Training and Evaluation," 2004.

${ }^{35}$ Sinacori, J. B., "The Determination of Some Requirements for a Helicopter Flight Research Simulation Facility," NASA Ames Research Center, 1097-1, Moffett Field, CA, 1977.

${ }^{36}$ Schroeder, J. A., "Helicopter Flight Simulation Motion Platform Requirements," NASA/TP-1999-208766, 1999.

${ }^{37}$ Mikula, J., Chung, W. W. Y., and Tran, D., "Motion Fidelity Criteria for Roll-Lateral Translational Tasks," AIAA Modeling and Simulation Technologies Conference, AIAA, 1999.

${ }^{38}$ Advani, S. K., Hosman, R. J. A. W., and Potter, M., "Objective Motion Fidelity Qualification in Flight Training Simulators," AIAA Modeling and Simulation Technologies Conference, AIAA, 2007.

${ }^{39}$ Evans, J. J., Wilson, B. A., Schuri, U., Andrade, J., Baddeley, A., Bruna, O., Canavan, T., Della Sala, S., Green, R., Laaksonen, R., Lorenzi, L., and Taussik, I., "A Comparison of 'Errorless' and 'Trial-and-Error' Learning Methods for Teaching Individuals with Acquired Memory Deficits," Neuropsychological Rehabilitation, Vol. 10, No. 1, 2000, pp. 67-101.

${ }^{40}$ Regional Airline Association (RAA), Government Research Indicates Simulator Motion Adds Training Complexity - RAA Recommends Operational Testing to Validate Effectiveness of Non-Motion Platforms, Regional Airline Industry White Paper, 2008.

${ }^{41}$ Bürki-Cohen, J., Soja, N. N., and Longridge, T., "Simulator Platform Motion--the Need Revisited," International Journal of Aviation Psychology, Vol. 8, No. 3, 1998, pp. 293-317.

${ }^{42}$ Delft University of Technology, "A Cybernetic Approach to Assess Simulator Fidelity," URL: http://www.lr.tudelft.nl/en/organisation/departments-and-chairs/control-and-operations/control-and-

simulation/backup/onderzoek/human-machine-systems-and-flight-simulation/cybernetic-approach-to-asses-simulator-fidelity/ [cited July 12, 2011].

${ }^{43}$ Steurs, M., Mulder, M., and van Paassen, R. M. M., "A Cybernetic Approach to Assess Flight Simulator Fidelity," AIAA Modeling and Simulation Technologies Conference, AIAA, 2004.

${ }^{44}$ Zaal, P. M. T., Pool, D. M., Mulder, M., and Van Paassen, M. M., "Multimodal Pilot Control Behavior in Combined Target-Following Disturbance-Rejection Tasks," AIAA Modeling and Simulation Technologies Conference, AIAA, 2009.

${ }^{45}$ Nieuwenhuizen, F. M., Zaal, P. M. T., Mulder, M., and Van Paassen, M. M., "A New Multi-Channel Pilot Model Identification Method for Use in Assessment of Simulator Fidelity," AIAA Modeling and Simulation Technologies Conference, AIAA, 2006.

${ }^{46}$ Pool, D. M., Zaal, P. M. T., Van Paassen, M. M., and Mulder, M., "Identification of Multimodal Pilot Models Using Ramp Target and Multisine Disturbance Signals," Journal of Guidance, Control, and Dynamics, Vol. 34, No. 1, 2011.

${ }^{47}$ van den Berg, P., Zaal, P. M. T., Mulder, M., and Van Paassen, M. M., "Conducting Multi-Modal Pilot Model Identification - Results of a Simulator Experiment," AIAA Modeling and Simulation Technologies Conference, AIAA, 2007.

${ }^{48}$ Smaili, H., Jansen, H., Naseri, A., Groen, E. L., and Stroosma, O., "Pilot Motion Perception and Control During a Simulated Decrab Maneuver," AIAA Modeling and Simulation Technologies Conference, AIAA, 2007.

${ }^{49}$ Groen, E. L., Smaili, M. H., and Hosman, R. J. A. W., "Simulated Decrab Maneuver: Evaluation with a Pilot Perception Model," AIAA Modeling and Simulation Technologies Conference, AIAA, 2005.

${ }^{50}$ Groen, E. L., Hosman, R. J. A. W., and Dominicus, J. W., "Motion Fidelity During a Simulated Takeoff," AIAA Modeling and Simulation Technologies Conference, AIAA, 2003.

${ }^{51}$ Nieuwenhuizen, F. M., Beykirch, K. A., Mulder, M., and Bülthoff, H. H., "Identification of Pilot Control Behavior in a Roll-Lateral Helicopter Hover Task," AIAA Modeling and Simulation Technologies Conference, AIAA, 2007.

${ }^{52}$ Berger, D. R., Terzibas, C., Beykirch, K., and Bülthoff, H. H., "The Role of Visual Cues and Whole-Body Rotation in Helicopter Hovering Control," AIAA Modeling and Simulation Technologies Conference, AIAA, 2007. 
${ }^{53}$ Beykirch, K., Nieuwenhuizen, F. M., Teufel, H. J., Nusseck, H.-G., Butler, J. S., and Bülthoff, H. H., "Control of a Lateral Helicopter Side-Step Maneuver in an Anthropomorphic Robot," AIAA Modeling and Simulation Technologies Conference, AIAA, 2007.

${ }^{54}$ Grant, P. R., Yam, B., Hosman, R., and Schroeder, J. A., "Effect of Simulator Motion on Pilot Behavior and Perception," Journal of Aircraft, Vol. 43, No. 6, 2006.

${ }^{55}$ Ellerbroek, J., Stroosma, O., Mulder, M., and van Paassen, M. M., "Identification of the Roles of Yaw and Sway Motion in Helicopter Yaw Control Tasks," AIAA Modeling and Simulation Technologies Conference, AIAA, 2007.

${ }^{56}$ Vaden, E. A., and Hall, S., "The Effect of Simulator Platform Motion on Pilot Training Transfer: A Meta-Analysis," International Journal of Aviation Psychology, Vol. 15, No. 4, 2005, pp. 375-393.

${ }^{57}$ van der Pal, J., "The Effect of Simulator Motion on Parameter Training for F-16 Pilots," Engineering Psychology and Cognitive Ergonomics: Transportation Systems, Medical Ergonomics and Training, edited by D. Harris, Ashgate, Oxford, England, 1999, pp. 267-275.

${ }^{58}$ De Winter, J. C. F., Dodou, D., and Mulder, M., "Training Effectiveness of Whole Body Flight Simulator Motion: A Comprehensive Meta-Analysis," International Journal of Aviation Psychology, in press.

${ }^{59}$ Cohen, J., Statistical Power Analysis for the Behavioral Sciences, 2 ed., Erlbaum, Hillsdale, NJ, 1988, pp. 1-552.

${ }^{60}$ Sparko, A. L., Bürki-Cohen, J., and Go, T. H., "Transfer of Training from a Full-Flight Simulator vs. a High Level Flight Training Device with a Dynamic Seat," AIAA Modeling and Simulation Technologies Conference, AIAA, 2010.

${ }^{61}$ Young, L. R., "Some Effects of Motion Cues on Manual Tracking," Journal of Spacecraft and Rockets, Vol. 4, No. 10, 1967, pp. 1300-1303. 